\title{
Neuromuscular Characteristics and Physical Function in Participants with Parkinson's Disease
}

Ji-Young Kim PhD ${ }^{1,2}$, Ji-Yong Byeon MS ${ }^{1}$, Hyuk-in Yang MS ${ }^{1}$, Jeonghoon Oh PhD ${ }^{3,4}$, Ju-Hee Lee PhD ${ }^{5}$, Moon-Ki Choi PhD ${ }^{6}$, Hae-Dong Lee $\mathrm{PhD}^{3}$, Justin Y Jeon $\mathrm{PhD}^{1,7,8}$

${ }^{1}$ Department of Sport Industry Studies, Yonsei University, Seoul; ${ }^{2}$ Physical activity and performance institute, Konkuk University, Seoul; ${ }^{3}$ Department of Physical Education, Yonsei University, Seoul, Korea; ' ${ }^{4}$ epartment of Neurosurgery, Houston Methodist Hospital, Houston, TX, USA; ${ }^{5}$ Mo-Im Kim Nursing Research Institute, College of Nursing, Yonsei University, Seoul; ${ }^{6}$ College of Nursing, Kangwon National University, Chuncheon; ${ }^{7}$ Cancer Prevention Center, Yonsei Cancer Hospital, Seoul; ${ }^{8}$ Exercise Medicine Center for Diabetes and Cancer Patients (ICONS), Seoul, Korea

PURPOSE: This study aimed to investigate the level of physical function, lower body strength, and muscle activation during various types of muscle contraction in participants with and without Parkinson's disease (PD).

METHODS: Twelve participants with PD (mean age $=63.17 \pm 6.24$ years) and 12 age- and sex-matched healthy adults (mean age $=$ $58.67 \pm 6.39$ years) were recruited. An isokinetic dynamometer was used to measure the length- and velocity-dependent maximum voluntary force and the rate of torque development (RTD) of the knee extensor muscles. Muscle activation of the vastus lateralis (VL), vastus medialis (VM), and rectus femoris (RF) muscles of both legs was examined using surface electromyography. The 6-minute walk test, chair stand test, timed up-and-go test, sit-and-reach test, and back-scratch test were performed to assess physical function.

RESULTS: Compared to healthy individuals, participants with PD showed significantly lower maximum voluntary force and RTD $(p<.05)$, performed fewer repetitions in the chair stand test $(11.64 \pm 1.75$ vs. $17.08 \pm 2.27, p<.001)$, were slower in the timed up-andgo test $(8.36 \pm 1.42$ vs. $5.65 \pm 1.07, p<.001)$, and walked shorter distances in the 6 -minute walk test $(424.17 \pm 65.97$ vs. $539.47 \pm 63.18$, $p<.001)$. However, activation of the three different muscles during isometric and isokinetic muscle contraction was not different between participants with and without PD.

CONCLUSIONS: Preserved muscle activation and significantly lower muscle strength during various types of muscle contractions may suggest lower muscle strength and efficiency. The lower physical function seen in participants with mild PD could be due to disease and low physical activity-related muscle atrophy rather than lower muscle activation.

Key words: Parkinson's disease, Muscle strength, Physical function, Maximum voluntary contraction

\section{INTRODUCTION}

Parkinson's disease (PD) is a chronic progressive disorder characterized by slowness of movement (bradykinesia), rigidity, tremor, and gait abnormalities [1,2]. The loss of dopamine (DA) and its pathway have been studied as the crucial neurobiological substrate of motor features in
PD [3]. According to the previous studies, the imbalance in multiple neurotransmitter pathways might lead to deficits in central motor drive and, it leads to aberrant recruitment of muscle fibers [4]. These central imbalances destabilize can cause the neuromuscular junction and, induce raising muscle fiber alteration with a sarcopenic-like phenotype [5].

Movement difficulties lead to decreased physical activity levels and

Corresponding author: Justin Y. Jeon Tel +82-2-2123-6197 Fax +82-2-2123-8648 E-mail jjeondyonsei.ac.kr

*This research was supported by Basic Science Research Program through the National Research Foundation of Korea (NRF) funded by the Ministry of Science, ICT and Future Planning (NRF-2012R1A1A3012645; NRF-2015R1A1A3A04001474).

*This research was also partially supported by the Yonsei University (Yonsei Signature Research Cluster Program 2021-0009).

Received 24 May 2021 Revised 15 Jul 2021 Accepted 16 Jul 2021

(a)This is an Open Access article distributed under the terms of the Creative Commons Attribution Non-Commercial License (https://creativecommons.org/licenses/by-nc/4.0/) which permits unrestricted non-commercial use, distribution, and reproduction in any medium, provided the original work is properly cited. 
impaired strength and physical function in patients with PD [6]. Patients with PD have difficulties in activities of daily living, especially due to muscle weakness [7]. Muscle weakness may lead to postural instability and difficulties in ambulation [8] and can be a secondary cause for bradykinesia in patients with PD [9]. Furthermore, lower extremity muscle strength is significantly correlated with clinical severity and the number of falls [10]. Falling is a serious problem experienced by a majority (68\%) of patients with PD [11]. According to Allen et al. [12], patients with PD who have low muscle power are 6 times more likely to report multiple falls than those who have high muscle power.

Patients with PD's muscle weakness seem to be accompanied by altered voluntary muscle activation [4], increased muscle co-activation and stiffness [13], and decreased rate of force development [14]. Furthermore, muscular weakness tends to exacerbate as the disease progresses [15], and therefore, patients with PD should be monitored constantly. Neuromuscular evaluation is important to assess performance-limiting factors, the effects of rehabilitation programs, and training intervention that limits the symptoms of the pathology [16].

Decreased muscle strength has been observed in patients with PD using an electromechanical dynamometer. Stevens-Lapsley et al. [4] measured the weakness, activation deficits, and fatigue in the quadriceps muscles of patients with PD and observed that patients with higher disease severity (Unified Parkinson's Disease Rating Scale [UPDRS] motor score $\geq 31.7$ ) had significantly more quadriceps weakness and central activation deficits than those with lower disease severity (UPDRS motor score $<31.7)$ or healthy controls. Durmus et al. [10] tested lower extremity isokinetic muscle strength in patients with PD and reported a significant decrease in isokinetic muscle strength, especially in the hip and knee flexors and extensors. In a study by Inkster et al. [17], reduced strength of the hip muscles (hip extensors) contributed to the difficulty experienced in standing up from a chair in patients with PD.

Parkinson's disease is a senile neurological disease caused by a lack of dopamine, in which the neuromuscular system and body functions decrease at the same time. However, limited studies have been conducted to assess neuromuscular characteristics of PD-related pathologic mechanisms and physical functions related to walking, standing up from a chair, and daily activities in patients with PD. Furthermore, limited studies have reported on muscle function and its relationship with physical function in patients with PD, such as muscle weakness, loss of ability to generate force, and general reconditioning, including electromyography (EMG) findings. Therefore, the purpose of the current study is to investigate the level of physical function, lower body strength, and muscle activation during various types of muscle contraction in participants with and without PD.

\section{METHODS}

\section{Participants}

A total of 12 participants with PD (4 males and 8 females; mean age $=63.17$ years $)$ and age- and sex-matched 12 healthy controls ( 3 males and 9 females; mean age $=58.67$ years) were recruited. Participants with PD were included if they 1) were $>40$ years old, 2) scored between 0 and 3 on the Hoehn and Yahr scale [18], 3) had no known injuries that might interfere with motor function, and 4) could walk independently without the aid of walking sticks. The control group included healthy participants who had no known neurological disorders or musculoskeletal injuries that might interfere with walking. This study was approved by the institutional review board at the Y University (IRB approval number: 201409-HRBR-234-03). All program administration and outcome evaluations were conducted after receiving written consents. Participants were provided with an explanation about the purpose of the study and procedures, and any questions that arose were answered. Participants were informed that they could withdraw from participation whenever they wanted. All participants signed an informed consent form before participating in the study.

\section{Anthropometric measurements}

Body weight and body composition including muscle mass, fat mass, and fat percentage were measured using bioelectrical impedance analysis (Inbody 720, Biospace, Korea).

\section{Maximum voluntary contraction (MVC)}

MVC of the knee extensor muscles and joint angle were measured using an isokinetic dynamometer (Con-Trex MJ, Physiomed, Switzerland). The participants' knee joints were aligned with the axis of the dynamometer and the trunk was secured using velcro straps across the waist and chest to prevent excessive movements. For all measurements, participants were seated with a hip joint angle of $80^{\circ}$. Maximum isometric contractions were performed at $60^{\circ}$ knee flexion $\left(0^{\circ}=\right.$ full knee extension). At this joint configuration, participants performed maximum isometric knee extension in two different ways: ballistic (ISO60Ball) (exert maximal torque as fast as possible) and ramp (ISO60Ramp) (gradually 
build up to maximal torque over 3 seconds). In the isokinetic concentric trials, knee extension was performed over the range of $0^{\circ}-110^{\circ}$ knee flexion and at angular speeds of $30 \% \mathrm{~s}(\mathrm{CON} 30)$ and $120 \% \mathrm{~s}(\mathrm{CON} 120)$.

During isometric and isokinetic contraction strength testing, participants were instructed to exert maximal voluntary knee extensions against the lever arm of the dynamometer. Strong verbal encouragement was given, and visual feedback about their torque was provided to provide motivation and consistency. A rest period of at least 2 minutes was given between each trial. Before each isometric and isokinetic concentric trials, participants performed familiarization trials with $70 \%-80 \%$ of MVC. None of the participants reported fatigue during the familiarization trials. The participants performed 2 repetitions of isometric ballistic (ISO60Ball) and ramp (ISO60Ramp) knee extension contraction and 3 repetitions of isokinetic knee extension at a velocity of 30\% (CON30) and $120 \%$ s (CON120) for both legs.

Peak torque of the isometric trial was determined by taking the highest torque value obtained during the $500-\mathrm{ms}$ plateau phases. The rate of torque development (RTD) was derived as the slope of the linear portion of torque-time curve. RTD was determined from the first derivative of the ascending portion of the torque-time curve and quantified from the linear slope of the torque-time curve at time intervals of 0-100 ms [19].

Muscle activation was measured using surface EMG electrodes (Trigno wireless EMG, DELSYS, Boston, MA) to assess the vastus lateralis $(\mathrm{VL})$, vastus medialis (VM), and rectus femoris (RF) muscles on both legs, as described by the SENIAM conventions (www.seniam.org). To reduce skin impedance, the skin locations where the electrodes were placed were shaved (if necessary), cleaned with ethanol cotton pads, and dried. EMG signals were amplified, band-pass filtered (20-450 Hz), and digitized with a sampling frequency of $1 \mathrm{kHz}$ using an analog-to-digital converter (NI 6036E, National Instruments, Austin, USA). The EMG and torque signals were sampled at $1 \mathrm{kHz}$ and stored on a hard drive. The signals were analyzed using Chart v 5.5 for windows (AD instruments Ltd, USA). The average of the root-mean-square (RMS) values were calculated for the VL, VM, and RF muscles during MVCs test. EMG signals of testing contraction were normalized to those of the reference isometric knee flexion at $60^{\circ}$.

\section{Physical function}

The 6-minutes walk test, chair stand test, timed up-and-go (TUG) test, sit-and-reach test, and back-scratch test were used to measure physical function $[20,21]$. For the 6-minutes walk test, participants were asked to walk as quickly as possible and cover the furthest distance possible in 6 min while walking. The distance covered was recorded in meters. For the chair stand test, participants were asked to stand up to a full standing position and then sit as many times as possible in 30 seconds without using arm support. For the TUG test, participants stood up from a chair, walked $3 \mathrm{~m}$, turned, returned to the chair, and sat down. The total time to completion was recorded to the nearest tenth of a second. For the sit-and-reach test, participants were instructed to sit close enough to the edge of the chair to be able to straighten one leg and the distance between extended fingers and the tip of their toe was recorded to the nearest half of $1 \mathrm{~cm}$. For the back-scratch test, participants were asked to bend one hand over the shoulder and the other hand on the middle of the back, with the fingers pointing toward each other, trying to make them overlap. The distance between the fingertips was recorded to the nearest half of $1 \mathrm{~cm}$.

\section{Statistical analysis}

All data were analyzed using SPSS 21.0 software (SPSS Inc., Chicago, IL, USA) and values were expressed as the mean \pm standard deviation (SD). The normality for continuous variables was determined by the Shapiro-Wilk test. Characteristics of the study participants were compared using the independent t-test for continuous outcomes, including age, weight, body mass index (BMI), muscle mass, fat mass, and fat percentage, and the chi-square test for categorical outcomes. Since the age of the participants with PD and the healthy control group was not similar, data analysis was performed by controlling for age and sex. Comparisons of muscle function and physical function between participants with PD and healthy controls were assessed using analysis of covariance (ANCOVA) after controlling for age and sex. $p$-values of $<.05$ were considered statistically significant. In addition, Spearman's correlation and partial correlation analyses were used to determine the potential associations between muscle function and physical function tests.

\section{RESULTS}

\section{Characteristics of participants}

A total of 24 participants (12 participants with PD and 12 healthy controls) participated in this study. The age and anthropometric characteristics of participants are described in Table 1. There was no significant difference in sex, age, weight, BMI, muscle mass, fat mass, fat percentage, and systolic and diastolic blood pressure between participants with and 
Table 1. Participant characteristics

\begin{tabular}{|c|c|c|c|c|c|}
\hline & \multicolumn{2}{|c|}{ Participants with PD $(n=12)$} & \multicolumn{2}{|c|}{ Healthy controls $(n=12)$} & \multirow{2}{*}{$p$-value } \\
\hline & Mean & SD & Mean & SD & \\
\hline Sex (male \%) & \multicolumn{2}{|c|}{$4(33.3)$} & \multicolumn{2}{|c|}{$3(25.0)$} & .653 \\
\hline Age (yr) & 63.17 & 6.24 & 58.67 & 6.39 & .095 \\
\hline Weight (kg) & 60.37 & 9.41 & 62.66 & 9.84 & .566 \\
\hline BMI & 23.56 & 2.41 & 24.94 & 2.54 & .874 \\
\hline Muscle mass (kg) & 23.76 & 5.68 & 24.11 & 5.00 & .437 \\
\hline Fat mass (kg) & 16.82 & 7.08 & 18.62 & 3.44 & .185 \\
\hline Percent fat (\%) & 27.83 & 10.43 & 29.88 & 4.16 & .537 \\
\hline Disease duration (yr) & 8.6 & 5.86 & NA & NA & NA \\
\hline \multirow[t]{2}{*}{ Hoehn and Yahr Scale (\%) } & 1 & $6(50)$ & NA & NA & NA \\
\hline & 2 & $6(50)$ & NA & NA & NA \\
\hline
\end{tabular}

BMI, Body mass index; NA, not applicable; PD, Parkinson's disease.

Significance at $p<.05$.

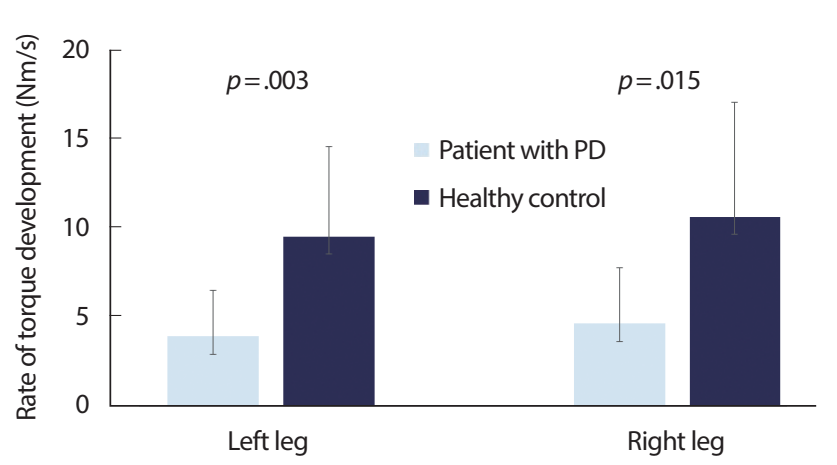

Fig. 1. Comparisons of the rate of torque development measured at 60deg flexed knee angle between participants with PD and healthy controls.

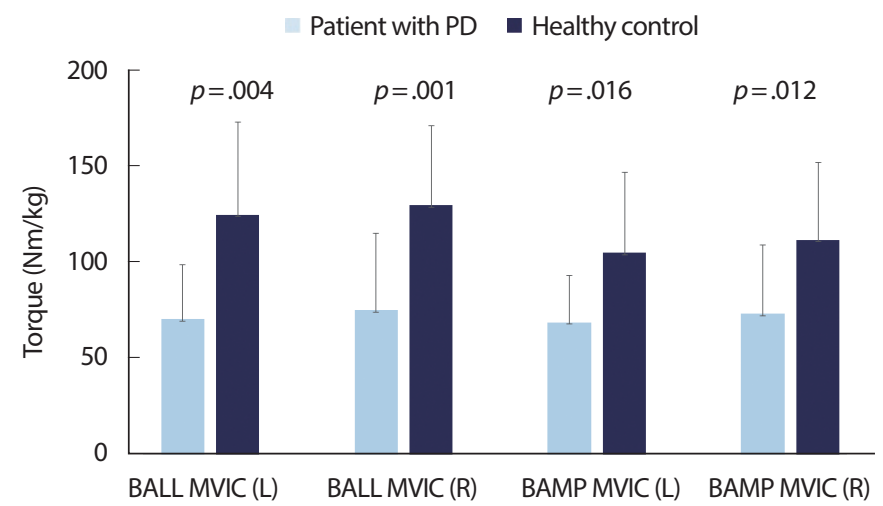

Fig. 2. Comparisons of peak torque achieved during ballistic (BALL MVIC) and ramp (RAMP MVIC) isometric contraction between participants with $\mathrm{PD}$ and healthy adults (Isometric measurements).

without PD.

\section{Comparisons of muscle function and electromyography findings between participants with and without Parkinson's disease}

Figs. 1-3 show the comparisons of RTD and peak torque between par-

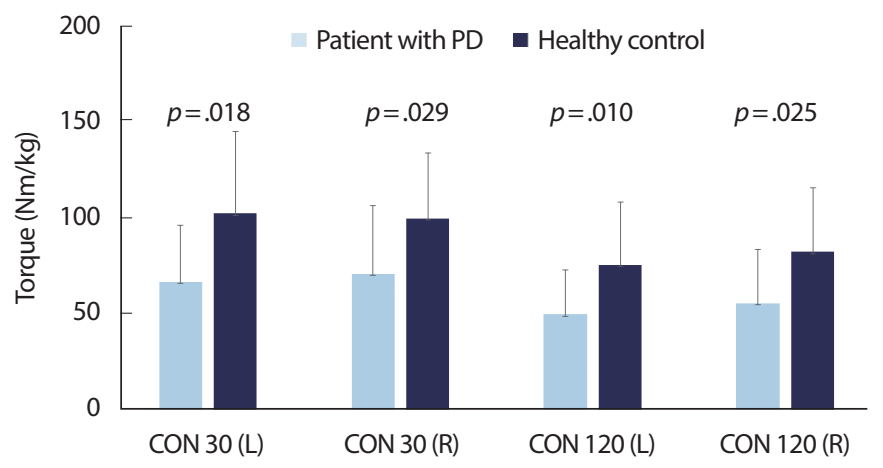

Fig. 3. Comparisons of peak torque obtained during slow ( $30 \mathrm{deg} / \mathrm{s}$ ) and fast (120 deg/s) maximum concentric knee extension between participants with PD and healthy adults (Isokinetic measurements).

ticipants with PD and healthy controls, respectively. RTD was significantly different between participants with PD and healthy controls (left side $3.94 \pm 2.57$ vs. $9.52 \pm 5.02, p=.003$; right side $4.66 \pm 3.14$ vs. $10.64 \pm$ $6.40, p=.015)$. Participants with PD had lower MVC torque value during isometric ballistic contraction (left side: $69.84 \pm 27.99$ vs. $123.78 \pm 47.87$, $p=.004$; right side: $74.56 \pm 39.39$ vs. $128.71 \pm 41.07, p=.001$ ), ramp contraction (left side: $68.22 \pm 24.33$ vs. $104.20 \pm 41.41, p=.016$; right side: $72.80 \pm 35.13$ vs. $110.74 \pm 39.85, p=.012$ ), and isokinetic contraction (left side at a velocity of $30 \%$ s: $66.43 \pm 29.12$ vs. $101.80 \pm 42.18, p=.018$; right side at a velocity of $30 \%$ s: $70.58 \pm 35.03$ vs. $99.23 \pm 33.98, p=.029$; left side at a velocity of $120^{\circ} / \mathrm{s} 49.69 \pm 22.64$ vs. $75.15 \pm 32.62, p=.010$; right side at a velocity of $120 \% 55.35 \pm 27.92$ vs. $81.88 \pm 33.26, p=.025)$ than healthy controls.

A comparison of the normalized RMS EMG values for various contractions between participants with Parkinson's disease and healthy controls did not reveal any differences, except for the VM in the right side isometric ballistic test at $60^{\circ}(p<.02)$ (Table 2). 
Table 2. Comparison of electromyography findings between patients with Parkinson's disease and healthy controls

\begin{tabular}{|c|c|c|c|c|c|}
\hline & \multicolumn{2}{|c|}{ Participants with PD $(n=12)$} & \multicolumn{2}{|c|}{ Healthy controls $(n=12)$} & \multirow{2}{*}{$p$-value } \\
\hline & Mean & SD & Mean & SD & \\
\hline \multicolumn{6}{|l|}{ EMG (\%) } \\
\hline \multicolumn{6}{|l|}{$\mathrm{VL}$} \\
\hline CON30 (L) & 166.59 & 91.63 & 145.26 & 38.21 & .226 \\
\hline CON30 (R) & 158.47 & 69.12 & 133.16 & 53.79 & .367 \\
\hline CON120 (L) & 193.51 & 96.50 & 167.39 & 50.50 & .062 \\
\hline CON120 (R) & 169.85 & 55.42 & 180.97 & 75.72 & .811 \\
\hline ISO60Ball (L) & 104.10 & 20.40 & 119.39 & 28.71 & .204 \\
\hline ISO60Ball (R) & 106.12 & 23.12 & 122.99 & 32.97 & .102 \\
\hline \multicolumn{6}{|l|}{ VM } \\
\hline CON30 (L) & 153.93 & 59.03 & 149.83 & 55.55 & .755 \\
\hline CON30 (R) & 142.68 & 34.18 & 142.39 & 59.54 & .839 \\
\hline CON120 (L) & 181.87 & 64.74 & 174.68 & 68.69 & .476 \\
\hline CON120 (R) & 176.48 & 51.07 & 196.77 & 92.95 & .592 \\
\hline ISO60Ball (L) & 106.30 & 41.12 & 114.55 & 34.63 & .495 \\
\hline ISO60Ball (R) & 94.76 & 18.12 & 121.51 & 35.68 & $.020^{*}$ \\
\hline \multicolumn{6}{|l|}{ RF } \\
\hline CON30 (L) & 167.45 & 67.54 & 163.58 & 63.02 & .590 \\
\hline CON30 (R) & 141.30 & 39.31 & 132.89 & 49.56 & .418 \\
\hline CON120 (L) & 210.74 & 104.88 & 179.56 & 59.50 & .101 \\
\hline CON120 (R) & 154.47 & 46.41 & 148.47 & 46.75 & .566 \\
\hline ISO60Ball (L) & 105.88 & 22.77 & 120.86 & 28.01 & .154 \\
\hline ISO60Ball (R) & 100.56 & 21.08 & 109.65 & 29.24 & .279 \\
\hline
\end{tabular}

Adjusted for age and sex.

EMG, electromyography; VL, vastus lateralis; VM, vastus medialis; RF, rectus femoris; CON30, isokinetic knee extension at 30\% $/ \mathrm{s}$; CON120, isokinetic knee extension at $120^{\circ} / \mathrm{s}$; ISO60Ball, ballistic isometric knee extension at $60^{\circ} ; \mathrm{PD}$, Parkinson's disease.

${ }^{*} p<.05$.

Table 3. Comparison of functional fitness between participants with and without PD

\begin{tabular}{|c|c|c|c|c|c|}
\hline & \multicolumn{2}{|c|}{ Participants with PD $(n=12)$} & \multicolumn{2}{|c|}{ Healthy controls $(n=12)$} & \multirow{2}{*}{$p$-value } \\
\hline & Mean & SD & Mean & SD & \\
\hline \multicolumn{6}{|l|}{ Flexibility (cm) } \\
\hline Sit \& reach $(\mathrm{L})$ & 6.33 & 8.67 & 12.96 & 8.33 & .255 \\
\hline Sit \& reach $(R)$ & 5.67 & 8.46 & 12.79 & 8.86 & .197 \\
\hline Back stretch $(\mathrm{L})$ & -14.42 & 6.46 & -12.21 & 10.74 & .803 \\
\hline Back stretch $(\mathrm{R})$ & -7.92 & 9.13 & -5.38 & 11.28 & 637 \\
\hline Chair stand (n) & 11.64 & 1.75 & 17.08 & 2.27 & $<.001^{* *}$ \\
\hline Timed up-and-go (s) & 8.36 & 1.42 & 5.65 & 1.07 & $<.001^{* *}$ \\
\hline 6-min walk (m) & 424.17 & 65.97 & 539.47 & 63.18 & $<.001^{* *}$ \\
\hline
\end{tabular}

Adjusted age and sex.

$P D$, Parkinson's disease; $L$, left; $R$, right.

${ }^{* *} p<.01$.

\section{Comparisons of physical function between participants with and without Parkinson's disease}

Table 3 shows the differences in flexibility and results of the chair stand test, the TUG test, and the 6-minute walk test between participants with and without PD. After adjusting for age and sex, participants with PD performed significantly worse than healthy controls on all physical function tests, except for flexibility. Compared to healthy controls, participants with PD performed fewer repetitions on the chair stand test (11.64 \pm 1.75 vs. $17.08 \pm 2.27, p<.001)$, were slower on the TUG test $(8.36 \pm 1.42$ vs. $5.65 \pm 1.07, p<.001)$, and walked shorter distances on the 6 -min walk test $(424.17 \pm 65.97$ vs. $539.47 \pm 63.18, p<.001)$. No significant differences in flexibility were found between participants with and without PD. 
Table 4. Relationships between muscle function and functional fitness tests

\begin{tabular}{|c|c|c|c|c|c|c|c|c|c|}
\hline & \multicolumn{3}{|c|}{ 6-min walk test } & \multicolumn{3}{|c|}{ Chair stand test } & \multicolumn{3}{|c|}{ Timed up-and-go test } \\
\hline & Model 1 & Model 2 & Model 3 & Model 1 & Model 2 & Model 3 & Model 1 & Model 2 & Model 3 \\
\hline RTD ISO60Ball (L) & $0.621^{* *}$ & $0.588^{* *}$ & $0.535^{*}$ & $0.660^{* *}$ & $0.482^{*}$ & 0.437 & $-.0718^{* *}$ & $-0.583^{* *}$ & $-0.583^{* *}$ \\
\hline RTD ISO60Ball (R) & $0.673^{* *}$ & $0.605^{* *}$ & $0.528^{*}$ & $0.752^{* *}$ & $0.559^{*}$ & $0.510^{*}$ & $-0.719 * *$ & $-0.525^{*}$ & $-0.533^{*}$ \\
\hline ISO60Ball (L) & $0.566^{* *}$ & $0.547^{*}$ & $0.459^{*}$ & $0.601^{* *}$ & $0.525^{*}$ & $0.471^{*}$ & $-0.632^{* *}$ & $-0.547^{*}$ & $-0.558^{*}$ \\
\hline ISO60Ball (R) & $0.649^{* *}$ & $0.598^{* *}$ & $0.507^{*}$ & $0.557^{* *}$ & $0.602^{* *}$ & $0.558^{*}$ & $-0.611^{* *}$ & $-0.568^{* *}$ & $-0.598^{* *}$ \\
\hline ISO60Ramp (L) & $0.551^{* *}$ & $0.568^{* *}$ & $0.453^{*}$ & $0.444^{*}$ & 0.395 & 0.299 & -0.384 & -0.401 & -0.436 \\
\hline ISO60Ramp (R) & $0.714^{* *}$ & $0.691^{* *}$ & $0.632^{* *}$ & $0.609^{* *}$ & $0.585^{* *}$ & $0.559^{*}$ & $-0.489^{*}$ & -0.403 & $-0.457^{*}$ \\
\hline CON30 (L) & $0.639^{* *}$ & $0.599 * *$ & $0.506^{*}$ & $0.527^{* *}$ & $0.495^{*}$ & 0.431 & $-0.522^{* *}$ & $-0.497^{*}$ & $-0.519^{*}$ \\
\hline CON30 (R) & $0.617^{* *}$ & $0.654^{* *}$ & $0.572^{* *}$ & $0.505^{*}$ & $0.620^{* *}$ & $0.585^{* *}$ & -0.399 & -0.41 & -0.425 \\
\hline CON120 (L) & $0.755^{* *}$ & $0.712^{* *}$ & $0.648^{* *}$ & $0.570^{* *}$ & $0.595^{* *}$ & $0.550^{*}$ & $-0.491 *$ & $-0.482^{*}$ & $-0.502^{*}$ \\
\hline CON120 (R) & $0.707^{* *}$ & $0.567^{* *}$ & $0.453^{*}$ & $0.613^{* *}$ & $0.475^{*}$ & 0.404 & $-0.530^{* *}$ & -0.344 & -0.354 \\
\hline
\end{tabular}

Data are presented as $r$ (correlation coefficient).

RTD, rate of force development; ISO60Ball, ballistic isometric knee extension at $60^{\circ}$; ISO60Ramp, ramp isometric knee extension at $60^{\circ}$; CON30, isokinetic knee extension at $30^{\circ} / \mathrm{s}$; CON120, isokinetic knee extension at $120^{\circ} / \mathrm{s}$; L, left; R, right.

Model 1: Crude. Model 2: Partial correlation between muscle function and functional fitness tests after adjusting for age and sex. Model 3: Partial correlation between muscle function and functional fitness tests after adjusting for age, sex, and weight.

${ }^{*} p<.05$ (two-tailed); ${ }^{* *} p<.01$ (two-tailed).

\section{Association between muscle function and physical function}

The results of the 6-minute walk test, the chair stand test, and the TUG test were associated with muscle function (Table 4). The results of the 6-minute walk test were associated with RTD and peak torque on ISO60Ball, ISO60Ramp, CON30, and CON120 on both legs, even after adjusting for age, sex, and weight. The chair stand test, a measure of lower muscle strength, showed a significant correlation with all muscle function parameters. However, the correlations failed to reach statistical significance after adjusting for age, sex, and weight. The TUG test, a measure of speed, agility, and balance, showed significant correlations with the rate of force development and peak torque of ISO60Ball on both legs, even after adjusting for age, sex, and weight.

\section{DISCUSSION}

This study investigated the neuro-muscular characteristics and physical function of participants with PD and compared them with those of age- and sex-matched healthy controls. The study results clearly demonstrate that, as hypothesized, participants with PD had significantly lower physical function. Coincide with the reduction in physical function, rate of torque development, peak torque during isometric and isokinetic contraction were all significantly decreased in participants with PD. However, muscle activation, measured by EMG were preserved among participants with PD. Since muscle strength could be determined by neural and muscular alteration due to PD, we can speculate that lower physical function observed among our participants were due to lower muscular alteration rather than neural factors. However, the reduced rate of torque development may suggest a contribution of the central nervous system (CNS) for reduced physical function and lower body strength in participants with PD.

This result is somewhat contradictory to a recently reported study which showed preserved skeletal muscle mass and force expression with significant reduction in neuromuscular activation among physically active participants with PD. Both studies reported a reduction in the rate of torque development in participants with PD, while different results were shown in maximum voluntary force: Our study showed significantly decreased maximum voluntary force in participants with PD while Martignon et al. showed no difference in maximal voluntary force between participants with and without PD [22]. Knowing that participants in the Martignon et al's study were physically active, who regularly exercise at the University affiliated hospital training center under the supervision of the certified therapist, discrepancy between two studies could be due to exercise participation.

Significantly reduced lower limb muscle strength in participants with PD compared to that in healthy controls as observed in our study is not surprising. Muscle weakness, which is a reduction in the amount of force caused by muscle contraction, has been previously reported in participants with PD [23]. Furthermore, muscle weakness has been investigated in overall muscle groups, including the trunk [24], upper limbs [7], and 
lower limbs $[10,25]$ in participants with PD. Our results show that participants with PD have lower torque output compared to healthy controls despite having similar muscle activation. Clark and Fielding reported a significant positive correlation between the rate of surface EMG rise in the quadriceps muscle group and power during a leg extension task in healthy older adults $(\mathrm{r}=0.61, p<.001)$ [26]. Thus, in our study, results showing similar muscle activation in sEMG between participants with PD and healthy controls, but significantly weaker muscle strength and physical function in participants with PD than in healthy control, was rather surprising. Since PD is a central nervous system disorder, identified as a lower dopamine level, we hypothesized that participants with PD will have significantly reduced muscle activation on EMG compared with healthy controls. There was no difference in EMG findings between participants with PD and healthy controls during MVC, although the reason for this is unclear. Time since diagnosis, individual characteristics, or medication use may influence sEMG findings. A possible explanation for this observation in our study would be that the impaired motor unit recruitment ability might have been compensated by an increase in muscle activation of the recruited motor unit, as shown by StevensonLapsley et al. [4]. In addition, dopaminergic drug administration in patients with PD modifies their electrophysiological and behavioral patterns, making them more similar to those of normal subjects by restoring the ability of such patients to use more automatic motor learning strategies [27]. Since we did not control dopaminergic drugs for the safety of the participants in this study might explain preserved muscle activation among our participants. Thus, further research is necessary to address this.

This study also shows that participants with PD had significantly lower physical function related to daily activities than age- and sex-matched healthy controls. In line with findings of previous studies, participants with PD in this study also struggled with sitting and standing from a chair, walking, and mobility compared to the healthy controls. Such functional tasks are physically demanding and required for independent living. Canning et al. observed that the participants in the PD group walked the lesser distance in the 6-minute walk test than those in the control group and that despite recording similar fast-as-possible walking velocity, participants in the PD group walked at only $76 \%$ of their fast-aspossible velocity during the 6-min walk test, whereas those in the control group walked at $84 \%$ of their fast-as-possible velocity [28]. In addition, Brod, Mendelsohn, and Roberts have reported that $81 \%$ of participants with PD have difficulty with standing up from a chair [29].
Previous studies have reported that leg strength, particularly extensor strength, is associated with chair-rising performance and functional mobility measured by the TUG test $[17,30]$. In addition, Falvo and Earhart have reported that the 6-min test results are affected in part by diseasespecific characteristics, including age, Hoehn and Yahr scale score, and UPDRS score, in participants with PD [31]. However, limited studies have been conducted to assess the association between leg strength and the 6-min walk test results. In this study, the correlation analysis showed that the 6-min walk test result is an adequate indirect marker of muscular function in participants with PD, even after adjusting for age, sex, and weight. Thus, the 6-min walk test is a safe and practical way to measure muscle fitness in participants with PD. It shows significant correlations with MVC and may be a valuable assessment tool.

Exercise is an effective direct method of treatment, which improves muscle strength and physical function in participants with PD. A metaanalysis has reported that aerobic exercise improves motor actions, balance, and gait in participants with PD [32]. Progressive resistance exercise also improves muscle size, muscle strength, muscle endurance, and neuromuscular function in participants with PD [23]. However, the optimal exercise prescription for individuals with PD is yet to be established. Hence, it is important to understand the muscular and functional characteristics of participants with PD to develop an evidence-based exercise program for them. Therefore, this study offers important evidence that can be used to understand the muscle characteristics of participants with PD to develop an evidence-based PD patient-specific exercise-program and monitor their progress.

This study has a few limitations. Because of concerns regarding the health of participants with PD, the medication could not be controlled during the day of testing. Participants with PD were encouraged to take their medication at the regular time on the day of testing. Furthermore, because of the physically demanding nature of the tests, participants with PD who participated in this study generally had minimally influenced motor function in their lower extremities, with Hoehn and Yahr scale scores between 1 and 2, which may limit the generalizability of the results to the PD population. Another limitation is younger age among participants without PD compared with participants with PD. Although this difference is not statistically significant and age was controlled in all our analyses, we could not fully eliminate the possibility that better physical function among participants without PD observed in our study could have been due to their younger age. 


\section{CONCLUSION}

In conclusion, participants with PD had lower muscular function and physical function than healthy controls with preserved muscle activation. Preserved muscle activation yet significantly lower muscle strength during various types of muscle contractions may suggest lower muscle strength and physical function was seen in participants with mild PD could be due to disease and physical activity-related muscle atrophy rather than lower muscle activation. Further randomized controlled studies are needed to clarify whether exercise training could improve muscle strength and physical function of individuals with PD.

\section{CONFLICT OF INTEREST}

All authors declare that there is no conflict of interest regarding the publication of this paper.

\section{AUTHOR CONTRIBUTIONS}

Conceptualization: JY Kim, JY Jeon; Data curation: JY Byeon, Hi Yang, J Oh, MK Choi; Formal analysis: JY Kim, J Oh; Funding acquisition: JH Lee, MK Choi; Methodology: JY Kim, JY Byeon, Hi Yang; Project administration: HD Lee, JY Jeon; Visualization: JY Byeon, Hi Yang; Writing-original draft: JY Kim, JY Byeon, Hi Yang, J Oh; Writing-review \& editing: JH Lee, HD Lee, JY Jeon.

\section{ORCID}

$\begin{array}{ll}\text { Ji Young Kim } & \text { https:/orcid.org/0000-0001-7643-6132 } \\ \text { Ji Yong Byeon } & \text { https://orcid.org/0000-0002-4684-1740 } \\ \text { Hyuk in Yang } & \text { https://orcid.org/0000-0003-0634-750X } \\ \text { Jeonghoon Oh } & \text { https://orcid.org/0000-0002-9185-7362 } \\ \text { Ju Hee Lee } & \text { https://orcid.org/0000-0002-2805-1622 } \\ \text { Moon Ki Choi } & \text { https://orcid.org/0000-0002-0733-1190 } \\ \text { Hae Dong Lee } & \text { https://orcid.org/0000-0002-0546-2584 } \\ \text { Justin Y Jeon } & \text { https://orcid.org/0000-0001-7978-4271 }\end{array}$

\section{REFERENCES}

1. Nutt JG, Wooten GF. Diagnosis and initial management of parkinson's disease. N Engl J Med. 2005;353(10):1021-7.
2. Dibble LE, Lange M. Predicting falls in individuals with parkinson disease: a reconsideration of clinical balance measures. J Neurol Phys Ther. 2006;30(2):60-7.

3. Kordower JH, Olanow CW, Dodiya HB, Chu Y, Beach TG, et al. Disease duration and the integrity of the nigrostriatal system in parkinson's disease. Brain. 2013;136(8):2419-31.

4. Stevens-Lapsley J, Kluger BM, Schenkman M. Quadriceps muscle weakness, activation deficits, and fatigue with parkinson disease. Neurorehabil Neural Repair. 2012;26(5):533-41.

5. Rudolf R, Khan MM, Labeit S, Deschenes MR. Degeneration of neuromuscular junction in age and dystrophy. Front Aging Neurosci. 2014;6:99.

6. Roeder L, Costello JT, Smith SS, Stewart IB, Kerr GK. Effects of resistance training on measures of muscular strength in people with parkinson's disease: a systematic review and meta-analysis. PLoS One. 2015;10(7):e0132135.

7. Koller W, Kase S. Muscle strength testing in parkinson's disease. Eur Neurol. 1986;25(2):130-3.

8. Nallegowda M, Singh U, Handa G, Khanna M, Wadhwa S, et al. Role of sensory input and muscle strength in maintenance of balance, gait, and posture in parkinson's disease: a pilot study. Am J Phys Med Rehabil. 2004;83(12):898-908.

9. Berardelli A, Rothwell JC, Thompson PD, Hallett M. Pathophysiology of bradykinesia in parkinson's disease. Brain. 2001;124(11):2131-46.

10. Durmus B, Baysal O, Altinayar S, Altay Z, Ersoy Y, et al. Lower extremity isokinetic muscle strength in patients with parkinson's disease. J Clin Neurosci. 2010;17(7):893-6.

11. Wood BH, Bilclough JA, Bowron A, Walker RW. Incidence and prediction of falls in parkinson's disease: a prospective multidisciplinary study. J Neurol Neurosurg Psychiatry. 2002;72(6):721-5.

12. Allen NE, Sherrington C, Canning CG, Fung VS. Reduced muscle power is associated with slower walking velocity and falls in people with Parkinson's disease. Parkinsonism Relat Disord. 2010;16(4):261-4

13. Latash ML. Muscle coactivation: definitions, mechanisms, and functions. J Neurophysiol. 2018;120(1):88-104.

14. Wilson JM, Thompson CK, McPherson LM, Zadikoff C, Heckman CJ, et al. Motor unit discharge variability is increased in mild-to-moderate parkinson's disease. Front Neurol. 2020;11:477.

15. Moreno Catala M, Woitalla D, Arampatzis A. Central factors explain muscle weakness in young fallers with parkinson's disease. Neurorehabil and Neural Repair. 2013;27(8):753-9. 
16. Bozic PR, Celik O, Uygur M, Knight CA, Jaric S. Evaluation of novel tests of neuromuscular function based on brief muscle actions. J Strength Cond Res. 2013;27(6):1568-78.

17. Inkster LM, Eng JJ, MacIntyre DL, Stoessl AJ. Leg muscle strength is reduced in parkinson's disease and relates to the ability to rise from a chair. Mov Disord. 2003;18(2):157-62.

18. Hoehn MM, Yahr MD. Parkinsonism: onset, progression and mortality. Neurology. 1967;17:427-42.

19. Andersen LL, Aagaard P. Influence of maximal muscle strength and intrinsic muscle contractile properties on contractile rate of force development. Eur J Appl Physiol. 2006;96(1):46-52.

20. Jones CJ, Rikli RE. Measuring functional. J Active Aging. 2001;1:2430.

21. Podsiadlo D, Richardson S. The timed "up \& go": A test of basic functional mobility for frail elderly persons. J Am Geriatr Soc. 1991;39(2): $142-8$.

22. Martignon C, Ruzzante F, Giuriato G, Laginestra FG, Pedrinolla A, et al. The key role of physical activity against the neuromuscular deterioration in patients with parkinson's disease. Acta Physiologica. 2021; 231(4):e13630.

23. David FJ, Rafferty MR, Robichaud JA, Prodoehl J, Kohrt WM, et al. Progressive resistance exercise and parkinson's disease: a review of potential mechanisms. Parkinsons Dis. 2012;124527.

24. Bridgewater KJ, Sharpe MH. Trunk muscle performance in early par- kinson's disease. Phys Ther. 1998;78(6):566-76

25. Allen NE, Canning CG, Sherrington C, Fung VS. Bradykinesia, muscle weakness and reduced muscle power in parkinson's disease. Mov Disord. 2009;24(9):1344-51.

26. Clark DJ, Fielding RA. Neuromuscular contributions to age-related weakness. J Gerontol A Bio Sci Med Sci. 2012;67(1):41-7.

27. Fattapposta F, Pierelli F, My F, Mostarda M, Del Monte S, et al. L-dopa effects on preprogramming and control activity in a skilled motor act in parkinson's disease. Clin Neurophysiol. 2002;113(2):243-53.

28. Canning CG, Ada L, Johnson JJ, McWhirter S. Walking capacity in mild to moderate parkinson's disease. Arch Phys Med Rehabil. 2016 87(3):371-5.

29. Brod M, Mendelsohn GA, Roberts B. Patients' experiences of parkinson's disease. J Gerontol B Psychol Sci Soc Sci. 1998;53(4):213-22.

30. Schilling BK, Karlage RE, LeDoux MS, Pfeiffer RF, Weiss LW, et al. Impaired leg extensor strength in individuals with parkinson disease and relatedness to functional mobility. Parkinsonism Relat Disord. 2009;15(10):776-80

31. Falvo MJ, Earhart GM. Six-minute walk distance in persons with parkinson disease: a hierarchical regression model. Arch Phys Med Rehabil. 2009;90(6):1004-8.

32. Shu HF, Yang T, Yu SX, Huang HD, Jiang LL, et al. Aerobic exercise for parkinson's disease: a systematic review and meta-analysis of randomized controlled trials. PLoS One. 2014;9(7):e100503. 